\title{
Nierenteilresektion bei cT1 Nierentumoren: Vergleich der konventionellen laparoskopischen Nierenteilresektion mit der LESS Nierenteilresektion in SITUS-Technik
}

\author{
Partial Nephrectomy in cT1 Renal Tumours: Conventional Laparoscopic Partial \\ Nephrectomy Compared with LESS Partial Nephrectomy in SITUS Technique
}

Autoren

Institute
M. Wolters ${ }^{1}$, L. Wohlatz ${ }^{1}$, T. Neumann ${ }^{1}$, S. Jutzi ${ }^{1}$, I. Peters ${ }^{1}$, C. von Klot ${ }^{1}$, F. Imkamp ${ }^{1}$, B. Lutze ${ }^{3}$ M. A. Kuczyk' ${ }^{1}$, U. Nagele' ${ }^{2}$, T. R. W. Herrmann ${ }^{1}$

Klinik für Urologie und Urologische Onkologie, Medizinische Hochschule Hannover, Hannover Klinik für Urologie und Andrologie, Landeskrankenhaus Hall in Tirol (LKH), Hall in Tirol, Österreich 3 Institut für Hygiene/Krankenhaushygiene, Universitätsklinikum Leipzig, Leipzig

\section{Schlüsselwörter}

- Laparoskopie

- Nierenteilresektion

- laparoendoscopic single site surgery (LESS)

- nephron sparing surgery (NSS)

- single incision transumbilical surgery (SITUS)

Key words

- laparoscopy

- partial nephrectomy

- laparoendoscopic single site surgery (LESS)

- single incision transumbilical surgery (SITUS)
Bibliografie

DOI http://dx.doi.org/ 10.1055/s-0041-106151

Online-Publikation: 17.11.2015

Akt Urol 2015; 46: 453-460

(c) Georg Thieme Verlag KG

Stuttgart · New York

ISSN 0001-7868

Korrespondenzadresse

PD Dr. Thomas Herrmann

Stellvertretender Klinikdirektor Klinik für Urologie und Urologische Onkologie

Medizinische Hochschule

Hannover

Carl-Neuberg-Straße 1

30625 Hannover

herrmann.thomas@mh-

hannover.de

\section{Zusammenfassung}

\section{$\nabla$}

Hintergrund: Die Nierenteilresektion ist Therapie der Wahl bei Nierentumoren im klinischen Stadium 1 (cT1). Als Standardtherapie gilt die offene Operation, der Anteil minimalinvasiver Verfahren, wie der laparoskopischen, der robotisch assistierten oder der laparoendoskopischen single site (LESS) partiellen Nephrektomie, hat in den letzten Jahren zugenommen.

Patienten/Material und Methoden: In dieser retrospektiven Studie wurden alle Patienten erfasst, bei denen aufgrund des Verdachts auf einen malignen Nierentumor zwischen Dezember 2008 und November 2013 eine laparoskopische Nierenteilresektion durchgeführt wurde. Die Operationen erfolgten entweder als konventionelle transperitoneale Nierenteilresektionen (cLPN) oder als LESS partielle Nephrektomien (LESS-PN) in SITUS-Technik (single incision trancumbilical surgery). Ziel unserer Studie ist ein Vergleich beider Patientengruppen bezüglich perioperativer Outcomeparameter wie Operationsdauer, Ischämiezeit, Komplikationen, Transfusionsbedarf, Konversionsraten, Veränderungen der Nierenfunktion sowie der Krankenhausverweildauer. Ergebnisse: Im Zeitraum der Studie wurden 85 laparoskopische Nierenteilresektionen durchgeführt (72 cLPN, 13 LESS-PN). Die durchschnittliche Tumorgröße betrug 2,68 $\pm 1,47 \mathrm{~cm}$ (cLPN) vs. 2,46 $\pm 1,11$ (LESS-PN). Die Operationsdauer lag im Mittel bei $175,17 \pm 50,026 \mathrm{~min}$ (cLPN) und $185,77 \pm 35,991 \mathrm{~min}$ (LESS-PN). 45 (62,5\%) Operationen (cLPN) vs. 10 (76,9\%) (LESS-PN) erfolgten ohne Ischämie. Bezüglich perioperativer Outcomeparameter konnten keine signifikanten Unterschiede zwischen beiden Gruppen festgestellt werden. Die Rate postoperativer Komplikationen (ClavienDindo $\geq 3$ ) lag bei 11,1\% (cLPN) vs. 7,7\% (LESS-PN). Schlussfolgerung: Die LESS-Nierenteilresektion in SITUS-Technik ist eine attraktive Alternative zur konventionellen laparoskopischen Nierenteilresektion und zur offenen partiellen Nephrektomie.

\section{Abstract}

$\nabla$

Background: Partial nephrectomy is the treatment of choice for clinical stage 1 renal tumours. Open partial nephrectomy is the standard operative technique. The use of minimally-invasive strategies such as laparoscopic, robot-assisted partial nephrectomy or laparoendoscopic single site (LESS) partial nephrectomy has increased in recent years.

Patients/Material and Methods: In this retrospective study, patients undergoing laparoscopic partial nephrectomy between December 2008 and November 2013 were evaluated. All patients presented with renal lesions suspicious for malignancy. Operations were performed as conventional laparoscopic transperitoneal partial nephrectomies (cLPN) or LESS partial nephrectomies (LESS-PN) in SITUS technique (single incision transumbilical surgery). The aim of the study was to compare perioperative outcome parameters such as duration of surgery, time of ischaemia, complications, need for transfusion, conversion rates, changes in renal function and duration of hospital stay in both groups.

Results: A total of 85 laparoscopic partial nephrectomies were performed in this study (72 cLPN and 13 LESS-PN). The average tumour size was $2.68 \pm 1.47 \mathrm{~cm}$ (CLPN) vs. $2.46 \pm 1.11 \mathrm{~cm}$ (LESS-PN). The mean duration of surgery was $175.17 \pm 50.026 \mathrm{~min}(\mathrm{cLPN})$ and $185.77 \pm 35.991 \mathrm{~min}$ (LESS-PN). 45 (62.5\%) operations (cLPN) vs. 10 (76.9\%) (LESS-PN) were performed in zero-ischaemia technique. There were no significant differences in perioperative outcome parameters between both groups. Postoperative complication rates (Clavien-Dindo $\geq 3$ ) were $11.1 \%$ (cLPN) vs. 7.7\% (LESS-PN).

Conclusions: LESS partial nephrectomy in SITUS technique is an attractive alternative to conventional laparoscopic and open partial nephrectomy. 


\section{Einleitung \\ $\nabla$}

Die zunehmende Verbreitung bildgebender Verfahren hat in den letzten Jahrzehnten zu einer Verbesserung der Früherkennung kleiner, meist asymptomatischer Nierentumore geführt. Der einzige kurative Therapieansatz maligner Nierentumore ist die operative Entfernung. Gemäß der aktuellen europäischen Leitlinien ist die Nierenteilresektion die Therapie der Wahl bei Nierentumoren bis zu einer Größe von $\leq 7 \mathrm{~cm}$ [1]. Prinzipiell kann die Operation offen-chirurgisch, laparoskopisch oder roboterassistiert erfolgen. Die offene Nierenteilresektion ist derzeit die Standardtherapie, der Anteil minimalinvasiver Eingriffe hat allerdings in den letzten Jahren zugenommen. Die elektive Nierenteilresektion hat in der Therapie von Tumoren des Stadiums T1 gleiche onkologische Ergebnisse gezeigt wie die radikale Nephrektomie [2,3] und auch die Komplikationsraten der laparoskopischen Nierenteilresektion liegen im Bereich der offenen Chirurgie [4,5].

Bei der laparoskopischen Nierenteilresektion handelt es sich um einen technisch anspruchsvollen Eingriff, welcher derzeit nur in Zentren durchgeführt wird. Die adäquate Hämostase, die Rekonstruktion des Nierenbeckens und der Verschluss des Nierenparenchyms erfordern ein hohes Maß an operativem Geschick [6, 7]. Aus diesen Gründen ist die laparoskopische Nierenteilresektion kein Standardeingriff [1].

Die als „laparoendoscopic single site surgery“ (LESS) bezeichnete Technik, ist eine Variation der konventionellen Laparoskopie. Sie beinhaltet die Durchführung der Operation über einen einzigen operativen Zugang, entweder im Bereich des Bauchnabels, pararektal oder lumbal. Ziele der LESS-Technik sind neben der Reduktion des Zugangstraumas, eine schnellere Rekonvaleszenz und bessere kosmetische Ergebnisse im Vergleich zur konven- tionellen laparoskopischen oder robotischen Technik [8]. LESS wird derzeit hauptsächlich mit der Verwendung sogenannter plattform-basierter „single port“ Systeme gleichgesetzt, bei denen alle Instrumente (zumeist 2 zwei Instrumente und eine Optik) durch eine einzige Schleuse in den Patienten eingeführt werden. Daraus resultierende Nachteile im Vergleich zur konventionellen Multiport-Laparoskopie, bestehen vor allem in der fehlenden Triangulation und der dadurch erschwerten Koordination der Instrumente sowie dem sogenannten „clashing“, d.h. der gegenseitigen Behinderung von Optik und Instrumenten, aufgrund des nahezu identen Drehpunktes im Trokar. Hier kommen vorgebogene oder aktiv flektierbare Instrumente zum Einsatz. Durch die fehlende Triangulation und ein erschwertes Handling (aufgrund einer zumeist ungewollten multiaxialen Mitbewegung) sind sie jedoch dem herkömmlichen laparoskopischen Setting unterlegen [9].

Im Jahre 2009 wurde das Konzept der LESS-Chirurgie in SITUSTechnik etabliert [10]. Grundpfeiler dieses Operationsprinzips sind der Zugang durch den Bauchnabel und die Triangulation der Instrumente. Bei der SITUS-Technik werden gerade Optiken und Instrumente unter Verwendung eines einzigen periumbilicalen Schnittes über 3 Trokare in der Bauchdecke platziert ( $\bullet$ Abb. 1). Durch die vorhandene Triangulation der Instrumente ermöglicht dieses Verfahren ein laparoskopisches Operieren unter den gewohnten Bedingungen ohne die erwähnten Nachteile der „single port“ LESS-Technik.

Bisher fanden SITUS-Verfahren Anwendung bei der Nephrektomie und Tumornephrektomie, bei der Nierenteilresektion, der Zystektomie und der Pyeloplastik [10-15].

Ziel dieser Studie ist ein Vergleich klinischer Outcomeparameter und Komplikationsraten zwischen der LESS-Nierenteilresektion in SITUS-Technik und der konventionellen laparoskopischen
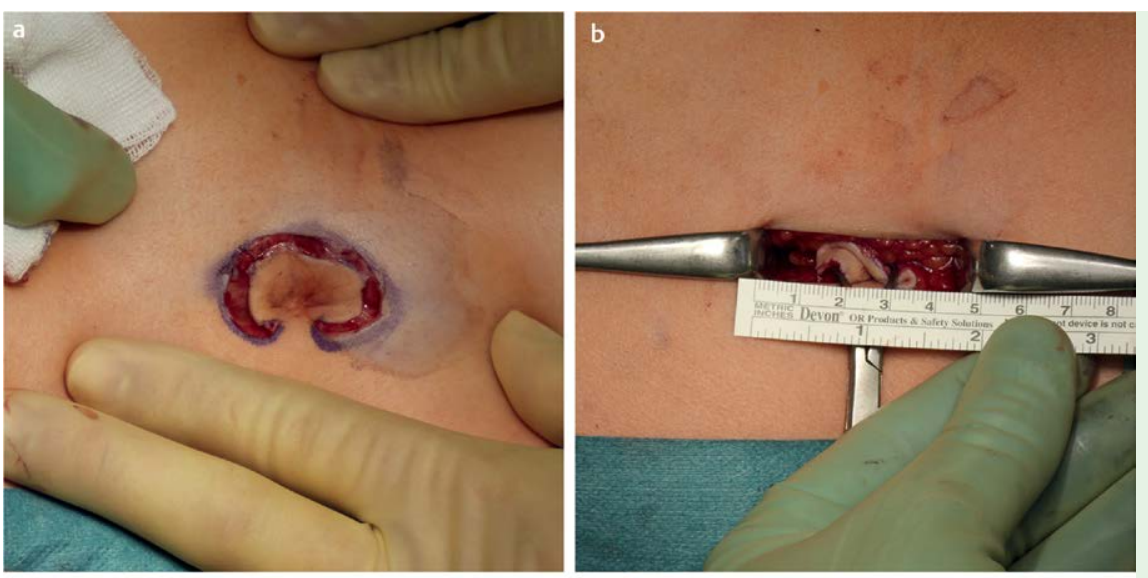

Abb. 1 Operativer Zugang LESS-PN.
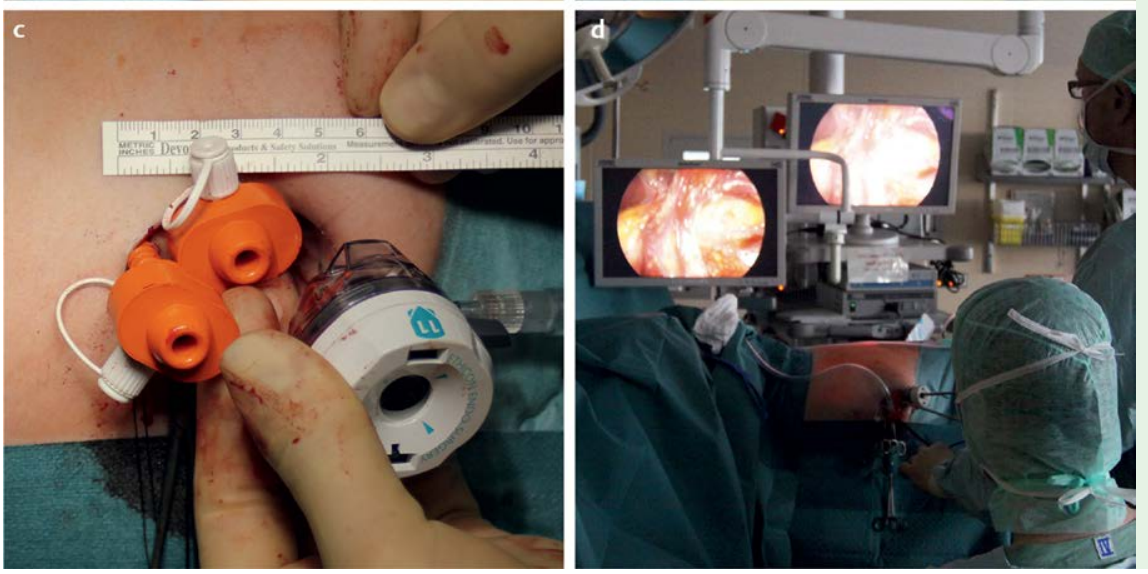
Multiport-Nierenteilresektion an der Medizinischen Hochschule Hannover, einem Schwerpunktzentrum für Nierenchirurgie.

\section{Patienten/Material und Methoden \\ $\nabla$}

In dieser retrospektiven Untersuchung wurden 85 Patienten erfasst, bei denen aufgrund des Verdachts auf ein cT1 Nierenzellkarzinom im Zeitraum Dezember 2008-November 2013 eine laparoskopische Nierenteilresektion durchgeführt wurde. Die Operationen erfolgten entweder in der konventionellen transperitonealen Multiport-Technik (cLPN) oder als LESS-partielle Nephrektomien (LESS-PN) in SITUS-Technik (single incision trancumbilical surgery). Während alle cLPN in der Medizinischen Hochschule Hannover (MHH) durchgeführt wurden, erfolgten 7 der 13 LESS-PN in Hall in Tirol (Österreich), wobei alle 13 Patienten vom selben Ärzteteam operiert wurden. Bei allen Patienten bestand präoperativ der Verdacht auf einen malignen Nierentumor (mindestens als Bosniak III Zyste klassifiziert) ( $\bullet$ Abb. 2). Die Läsionen wurden anhand der präoperativen Bildgebung bestehend aus CT oder MRT gemäß PADUA- und RENALScore klassifiziert [16,17].

\section{Datenanalyse}

Folgende demografische Parameter wurden erfasst: Alter, Geschlecht, Body-Mass-Index, Seite der betroffenen Niere, ASAScore, Charlson Komorbiditätsindex, abdominelle Voroperationen. Des Weiteren wurden Tumorcharakteristika wie Größe, Anzahl, PADUA und RENAL Score, histologische Befunde und das TNM-Stadium ausgewertet. Zusätzlich wurden folgende perioperative Parameter dokumentiert: Hämoglobin, Serumkreatinin, glomeruläre Filtrationsrate (GFR, berechnet nach CKD-EPI-
Formel; jeweils präoperativ und vor Entlassung), Operationsdauer (min), Ischämie vs. Zero-Ischämie, Ischämiezeit (min), intra- und postoperative Komplikationen (Clavien-Dindo-Klassifikation) $[18,19]$, Transfusionen, Konversionsraten und die Krankenhausverweildauer.

Die Ergebnisse sind als Mittelwerte mit Standardabweichung dargestellt, sowie als prozentuale Häufigkeiten. Stetige Variablen wurden als Mittelwert (Standardabweichung) angegeben. Der T-Test und wurde verwendet um stetige Variablen zu vergleichen, der Pearson Chi-Quadrat-Test wurde bei kategorialen Variablen angewendet. Alle p-Werte wurden 2-seitig geprüft, Signifikanz wurde angenommen bei $\mathrm{p}<0,05$.

\section{Operationstechnik}

Konventionelle transperitoneale laparoskopische Multiport-Nierenteilresektion (cLPN)

Nach Einlage eines transurethralen Dauerkatheters und $70^{\circ}$-Seitlagerung des Patienten wurde in Hassan-Technik ein ca. $2 \mathrm{~cm}$ langer pararektaler Zugang für die Kameraoptik etabliert. 2 weitere Inzisionen 2 Querfinger kaudal des Rippenbogens in der Medioclavicularlinie und 2 Querfinger mediokranial der Spina iliaca anterior superior wurden angelegt um einen $5 \mathrm{~mm} \mathrm{Ar}$ beitstrokar (Oberbauch) und einen $12 \mathrm{~mm}$ Trokar (Unterbauch) zu platzieren ( $\bullet$ Abb. 3$)$. Im Bedarfsfall wurde zusätzlich ein weiterer Port im Oberbauch platziert. Nach Insufflation des Abdomens mit 12-14 mmHg wurde der Situs inspiziert. Nach Lösung des Colons erfolgt die Identifikation des Harnleiters sowie der Gonadalgefäße. Aszendierend wurde der Unterpol in der Fettkapsel mobilisiert. Entlang der Gonadalvene wurde zum Hilus vorpräpariert. Es erfolgte eine subtile Darstellung der Hilusgefäße als Vorbereitung für etwaige temporäre Ischämie mithilfe Bulldog-Klemmung. Danach erfolgte die Eröffnung der Gerota
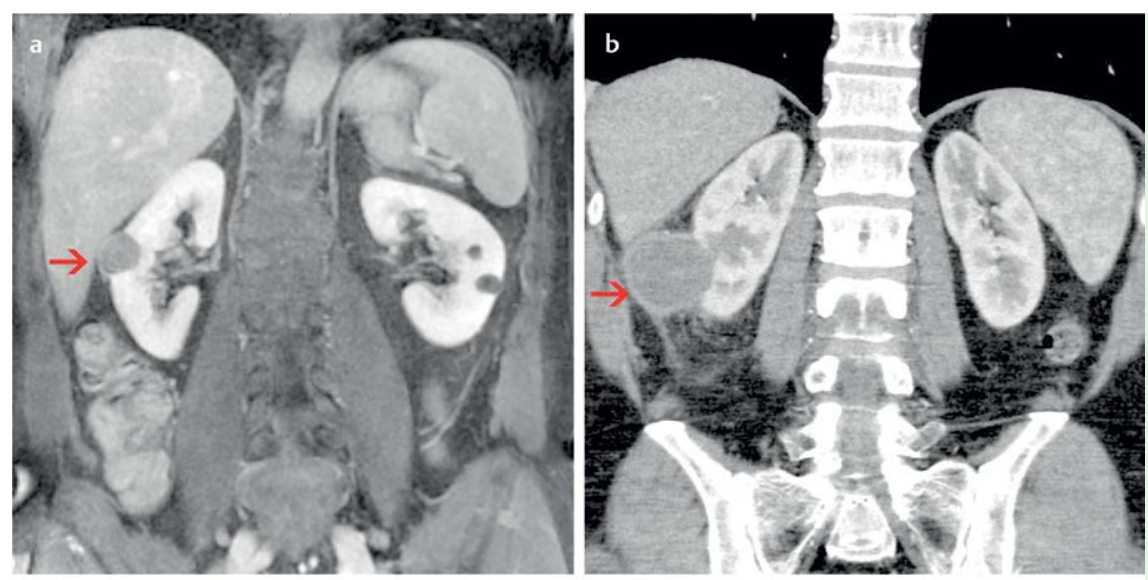

Abb. 2 Präoperative Bildgebung.
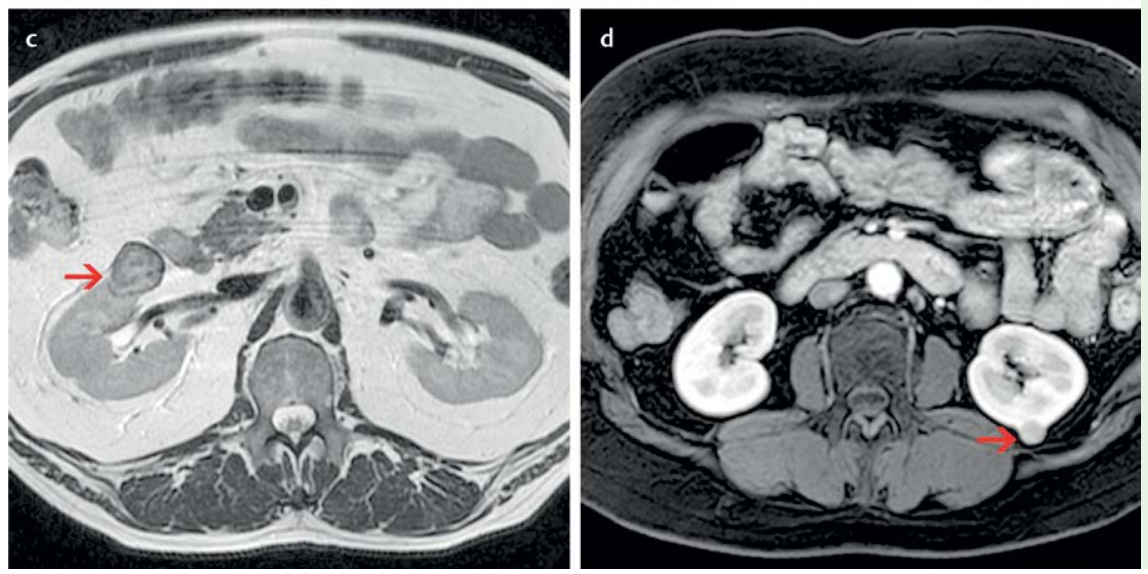

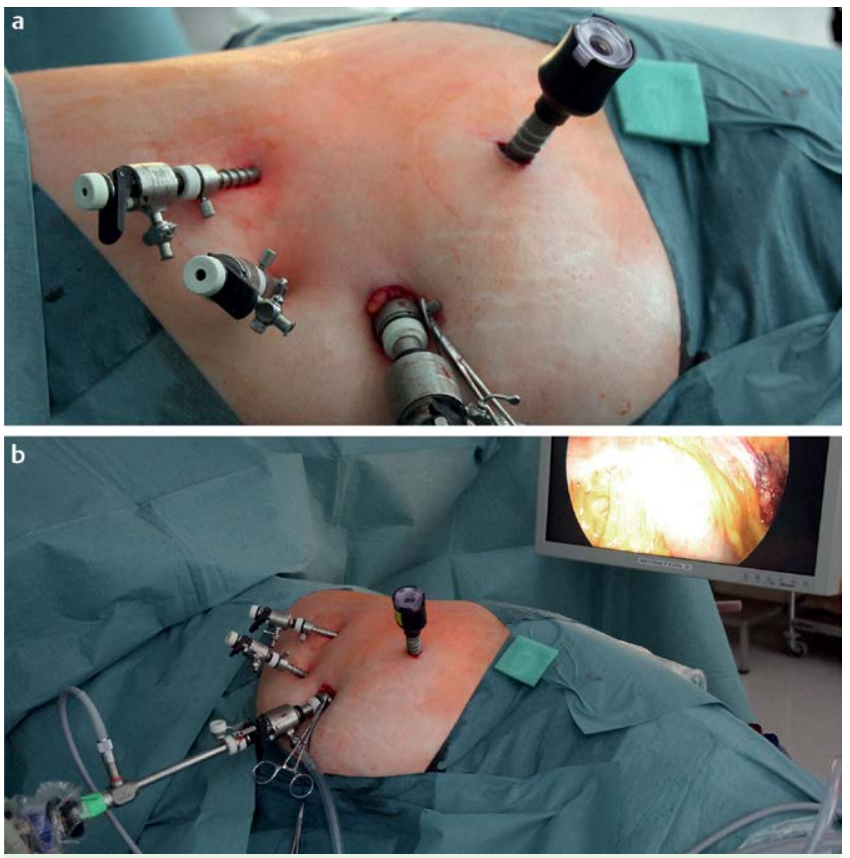

Abb. 3 Operativer Zugang cLPN.

Faszie auf der Nierenvorderfläche und Auslösen der Niere aus der perirenalen Fettkapsel.

Verwendet wurden herkömmliche 43-cm lange LaparoskopieInstrumente: Metzenbaumschere (Karl Storz, Tuttlingen, Deutschland), eine fenestrierte bipolare Koagulationspinzette (Aesculap, Braun, Tuttlingen, Deutschland), der En-Seal pressure coagulator und dissector (EnSeal NSEAL 535 RE, Ethicon, Hamburg, Deutschland), die Croce-Olmi-Fasszange (Karl Storz, Tuttlingen, Deutschland), der Hem-O-Lock Klammer Applikator XL (Weck, TeleXex, die USA), das „Elefant“ suction-system (Coloplast, Hamburg, Deutschland), und eine $10 \mathrm{~mm} 30^{\circ}$ Optik (Karl Storz, Tuttlingen, Deutschland) sowie ein HD-Kamera-System (Karl Storz, Tuttlingen, Deutschland).

Für die Leberretraktion wurde in der Regel ein $2 \mathrm{~mm}$ „needlescopic“ Instrument (Karl Storz, Tuttlingen, Deutschland) benutzt. Alternativ wurde die Leber auch mithilfe eines Mouret Nadelretraktors (Karl Storz, Tuttlingen, Deutschland) retrahiert.

\section{Hämostase}

Wenn möglich wurde eine vollständige Klemmung des arteriellen Gefäßes vermieden oder eine selektive Klemmung der Segmentarterien nach hilärer Präparation durchgeführt. Zur abklemmungsfreien Nierenteilresektion wurde der Tumor je nach Durchmesser mit 3 oder 4 transparenchymatösen Kompressionsnähten (2.0 Vicryl PCL 78) unterstochen, die beidseits mit Hem-o-Lock Clips gesichert wurden. Schließlich folgte die Exzision des Tumors mit der Metzenbaumschere gefolgt von Blutstillung durch bipolare Koaglation. Der Schnittrand wurde hierbei genau inspiziert um Biopsien des Tumorgrundes zu vermeiden. Wenn nötig wurde zur zusätzlichen Hämostase im Sinne einer Suture-Bolster-Naht die Entnahmestelle in üblicher Technik verschlossen.

Die Organbergung erfolgte mit einem Endo Catch I Bergebeutel (Autosuture, Covidien, Neustadt, Deutschland).
LESS partielle Nierenteilresektion in SITUS-Technik (LESS$\mathrm{PN})$

Die OP-Vorbereitung und Lagerung wurde analog zur konventionellen transperitonealen laparoskopischen Nierenteilresektion durchgeführt. Die Hautinzision erfolgte in Form einer plastischen c-förmigen Umschneidung des Nabels in der Umbilicalfalte ( $\bullet$ Abb. 1a). Nun wurde das subcutane Fettgewebe mobilisiert und die Rektusfaszie inzidiert. Nach Anlage von Faszienhaltenähten (Vicryl 2-0 TN MIC 514 Ethicon Hamburg, Deutschland) und Erzeugung eines Pneumoperitoneums in Verres-Technik, wurde ein $5 \mathrm{~mm}$ Kameratrokar (Karl Storz, Tuttlingen, Deutschland) in der Paramedianlinie seitlich des Nabels eingeführt. Mithilfe zweier Langenbeck-Haken konnte der Hautschnitt zu einer $4-7 \mathrm{~cm}$ messenden Längsinzision gedehnt werden ( $\bullet$ Abb. 1b). Dann wurden ein $5 \mathrm{~mm}$ Arbeitstrokar mit Insufflator (Karl Storz, Tuttlingen, Deutschland) und ca. $5 \mathrm{~cm}$ kaudal davon ein $11 \mathrm{~mm}$ Trokar (Endopath, Ethicon, Hamburg, Deutschland) durch den periumbilikalen Zugang in die Bauchhöhle eingebracht und durch Haltnähte gesichert ( $\bullet$ Abb. 1c/d). Nach Insufflation des Abdomens mit 12-14 mmHg folgte die Insepktion des Situs. Das darauffolgende intraoperative Vorgehen ist identisch mit dem der konventionellen laparoskopischen Nierenteilresektion. Nach der Tumorexzision und sorgfältiger Blutstillung, erfolgte die Entfernung des kaudalen Arbeitstrokars und das Einbringen des Endo Catch I Bergebeutels (Autosuture, Covidien, Neustadt, Deutschland) über diesen Zugang. Der Tumor wurde in den Bergebeutel verbracht und dieser nach Verbindung der Trokarinzisionen (abhängig von der Tumorgröße) unter Kamerakontrolle entfernt.

Verwendet wurden herkömmliche 43-cm lange LaparoskopieInstrumente: Metzenbaum Schere (Karl Storz, Tuttlingen, Deutschland), eine fenestrierte bipolare Koagulationspinzette (Aesculap, Braun, Tuttlingen, Deutschland), der En-Seal pressure coagulator und dissector (EnSeal NSEAL 535 RE, Ethicon, Hamburg, Deutschland), die Croce-Olmi-Fasszange (Karl Storz, Tuttlingen, Deutschland), der Hem-O-Lock Klammer Applikator XL (Weck, TeleXex, die USA), das „Elefant“ suction-system (Coloplast, Hamburg, Deutschland), und eine $50 \mathrm{~cm}$ lange $30^{\circ} 5 \mathrm{~mm}$ Optik (Karl Storz, Tuttlingen, Deutschland) sowie ein HD-KameraSystem (Karl Storz, Tuttlingen, Deutschland).

Nach Einlage einer Zieldrainage (15 CH Redon Drainage) im Bereich des Zugangs wurde die Hautinzision intrakutan mit resorbierbarem Nahtmaterial verschlossen.

\section{Ergebnisse}

$\nabla$

\section{Patienten}

Insgesamt wurden im Zeitraum der Studie 85 laparoskopische Nierenteilresektionen durchgeführt, wobei 72 Operationen $(84,7 \%)$ als konventionelle Nierenteilresektionen (Gruppe 1, cLPN) und 13 Eingriffe (15,3\%) als LESS-Nierenteilresektionen (Gruppe 2, LESS-PN) erfolgten. Hinsichtlich präoperativer Patienten- und Tumorcharakteristika zeigten sich keine signifikanten Unterschiede zwischen beiden Gruppen wie $\odot$ Tab. 1 demonstriert. Die mittleren Tumorgröße betrug 2,68 $\pm 1,47 \mathrm{~cm}$ in Gruppe 1 vs. 2,46 $\pm 1,11$ in Gruppe 2. PADUA und RENAL Score zeigten ebenfalls keine signifikanten Unterschiede im Vergleich beider Patientengruppen (PADUA Score 6,86 $\pm 0,89$ (Gruppe 1) vs. 7,46 $\pm 0,97$ (Gruppe 2); RENAL Score 5,17 $\pm 1,340$ (Gruppe 1) vs. $5,62 \pm 1,710$ (Gruppe 2)). Es zeigte sich, dass mehrheitlich Tumoren geringer Komplexität laparoskopisch operiert wurden. 
Tab 1 Preoperative Patienten- und Tumorcharakteristika.

\begin{tabular}{|c|c|c|c|}
\hline Variable & $\begin{array}{l}\text { Gruppe } 1 \\
\text { (cLPN) }\end{array}$ & $\begin{array}{l}\text { Gruppe } 2 \\
\text { (LESS-PN) }\end{array}$ & p-Wert \\
\hline Patienten, n (\%) & $72(84,7)$ & $13(15,3)$ & \\
\hline Alter (MW, SD) & $62,26(12,65)$ & $57,31(12,85)$ & 0.198 \\
\hline BMI, MW (SD) & $27,53(4,59)$ & $28,05(4,92)$ & 0.706 \\
\hline $\begin{array}{l}\text { Geschlecht } \\
\text { männl., n (\%) } \\
\text { weibl., n (\%) }\end{array}$ & $\begin{array}{l}57(79,2) \\
15(20,8)\end{array}$ & $\begin{array}{l}8(61,5) \\
5(38,5)\end{array}$ & 0.153 \\
\hline $\begin{array}{l}\text { Seite } \\
\text { rechts, n (\%) } \\
\text { links, n (\%) }\end{array}$ & $\begin{array}{l}26(36,1) \\
46(63,9)\end{array}$ & $\begin{array}{l}6(46,2) \\
7(53,8)\end{array}$ & 0.348 \\
\hline ASA-Score & $2,06(0,48)$ & $2,17(0,39)$ & 0.486 \\
\hline Charlson Komorbiditätsindex & $5,17(2,18)$ & $3,00(3,10)$ & 0.004 \\
\hline Abd. Vor-Ops, n (\%) & $22(30,6)$ & $8(61,5)$ & 0.035 \\
\hline Tumorgröße (cm), MW (SD) & $2,68(1,47)$ & $2,46(1,11)$ & 0.607 \\
\hline Tumoranzahl, MW (SD) & $1,00(0,00)$ & $1,154(0,554)^{*}$ & \\
\hline PADUA-Score & $6,86(0,89)$ & $7,46(0,97)$ & 0.031 \\
\hline RENAL-Score & $5,17(1,340)$ & $5,62(1,710)$ & 0.303 \\
\hline $\begin{array}{l}\text { Präop. Hämoglobin (g/dl), } \\
\text { (MW/SD) }\end{array}$ & $14,44(1,38)$ & $14,14(1,63)$ & 0.478 \\
\hline $\begin{array}{l}\text { Präop. Serumkreatinin } \\
(\mu \mathrm{mol} / \mathrm{I}),(n=84)\end{array}$ & $84,38(19,33)$ & $79,69(17,23)$ & 0.415 \\
\hline $\begin{array}{l}\text { Präop GFR (ml/min } / 1,73 \mathrm{~m}^{2} \text {, } \\
\text { CKD-EPI-Formel) }\end{array}$ & $82,13(18,62)$ & $84,37(20,62)$ & $0.6,96$ \\
\hline
\end{tabular}

30,6\% der Patienten die eine konventionelle Nierenteilresektion erhielten, wiesen abdominelle Voroperationen auf, gegenüber 61,5\% der Patienten mit einer LESS-Nierenteilresektion. Laborchemisch bestanden ebenfalls keine signifikanten Unterschiede zwischen beiden Gruppen ( $\bullet$ Tab. 1).

\section{Histologie}

Die histologische Untersuchung ergab bei 39 (54,2\%) Patienten aus Gruppe 1 und bei 9 (69,2\%) Patienten aus Gruppe 2 postoperativ einen malignen Befund ( $\bullet$ Tab. 2). Bei den 38 Patienten der Gruppe 1 mit der Diagnose eines Nierenzellkarzinoms zeigten sich in $92,1 \%$ der Fälle tumorfreie Schnittränder $(R=0)$, bei einem Patient wurde ein RX-Stadium dokumentiert und bei $2 \mathrm{~Pa}-$ tienten konnten die Tumore aufgrund von Quetschungsartefakten oder Zerbrechen im Bergebeutel nicht hinreichend beurteilt werden. In allen 3 Fällen konnte klinisch von einem R0-Stadium ausgegangen werden. Alle 9 Nierenzellkarzinome der Gruppe 2 wurden mit negativen Schnitträndern (R0-Stadium) reseziert.

\section{Operation}

Die Operationsdauer betrug im Mittel $175,17 \pm 50,026$ min für die konventionellen Nierenteilresektionen und $185,77 \pm 35,991$ min für die LESS-Eingriffe.

\section{Ischämie}

In der Gruppe der konventionellen Nierenteilresektionen konnten 45 (62,5\%) Operationen ohne Klemmung des Nierenhilus (Zero-Ischämie) erfolgen, 10 der 13 LESS-Nierenteilresektionen (76,9\%) waren ohne Ischämie durchführbar. Bei Betrachtung der Operationen, die eine Klemmung des Nierenhilus erforderten, betrug die mittlere Ischämiezeit 19,33 $\pm 8,42 \mathrm{~min}$ (Gruppe 1, $\mathrm{n}=27$ ) vs. $22,33 \pm 9,29 \mathrm{~min}$ (Gruppe $2, \mathrm{n}=3$ ).
Komplikationen

Bei 4 Patienten (5,6\%) der Gruppe 1 wurden intraoperative Komplikationen verzeichnet (eine Leberlazeration bei Tumoradhärenz am Oberpol mit Notwendigkeit einer Bluttransfusion und intensivmedizinischer Überwachung, eine kurzstreckige Deserosierung des Duodenums, welche zur Konversion auf eine offen-lumbale Nierenteilresektion führte und eine akzidentelle Inzision in den Nierentumor mit nachfolgender Konversion. Ein Patient erhielt aufgrund eines Pneumothorax eine Klammer Thoraxdrainage). Bei einem der 13 Patienten $(7,7 \%)$ die in LESSTechnik operiert wurden kam es intraoperativ zu einer Verletzung der Nierenvene, die rekonstruiert werden konnte.

Postoperative Komplikationen wurden bei insgesamt 8 Patienten der Gruppe 1 (11,1\%) dokumentiert. Die Art der Komplikationen (Clavien-Dindo $\geq 3$ ) zeigt $\odot$ Tab. 2. In Gruppe 2 traten keine signifikanten postoperativen Komplikationen auf.

\section{Konversion}

In 7 Fällen $(9,72 \%)$ war eine Konversion von der konventionellen Nierenteilresektion zur offenen Operation notwendig. Gründe waren die oben beschriebene Deserosierung des Duodenums, die reserosiert wurde und folgenlos blieb, ausgeprägte Verwachsungen und ungünstige Tumorlokalisationen, die eine sichere Tumorexzision erschwerten, sowie eine akzidentelle Inzision in den Nierentumor. In Gruppe 2 gab es keine Konversionen zum offenen Vorgehen. Lediglich eine Operation wurde durch das Einbringen zusätzlicher Trokare zur konventionellen Laparoskopie konvertiert. Grund war eine Blutung der Nierenvene, die problemlos laparoskopisch versorgt werden konnte.

\section{Transfusion}

Die Transfusionsrate der konventionell laparoskopisch operierten Patienten betrug 2,4\%. In Gruppe 2 bestand bei keinem Patienten die Notwendigkeit einer Bluttransfusion. Bei der letzten postoperativen Laborkontrolle vor der Entlassung zeigte sich in Gruppe 1 ein mittlerer Abfall des Hämoglobin-Wertes von $1,886 \pm 1,205 \mathrm{~g} / \mathrm{dl}$ gegenüber dem präoperativen Wert vs. $2,22 \pm 1,16 \mathrm{~g} / \mathrm{dl}$ in Gruppe 2 .

\section{Nierenfunktion}

Bei der Betrachtung der Retentionsparameter zeigte sich ein Abfall der Werte für die Glomeruläre Filtrationsrate (GFR, berechnet nach CKD-EPI-Formel) von 3,30 $\pm 10,667 \mathrm{ml} / \mathrm{min} / 1,73 \mathrm{~m}^{2}$ in Gruppe 1 vs. $5,81 \pm 16,829 \mathrm{ml} / \mathrm{min} / 1,73 \mathrm{~m}^{2}$ in Gruppe 2 (Vergleich der GFR präoperativ und vor Entlassung). Es bestand kein signifikanter Unterschied zwischen den Werten beider Gruppen (๑ Tab. 2).

\section{Krankenhausverweildauer}

Die stationäre Aufenthaltsdauer betrug 7,83 $\pm 2,638$ Tage (Gruppe 1 ) vs. 6,08 $\pm 1,656$ Tage (Gruppe 2).

\section{Diskussion}

Die Nierenteilresektion ist gemäß der europäischen Leitlinien für Urologie die Methode der Wahl bei Nierentumoren im klinischen Stadium 1 (cT1). Die Vorteile der nierenerhaltenenden Chirurgie sind gut durch Studien belegt $[1,20]$. Bei der Wahl des operativen Zugangs ist neben der Größe und der Lage des Tumors auch die Erfahrung des Chirurgen von Bedeutung. Unser Vergleich der konventionellen laparoskopischen Nierenteil- 
Tab. 2 Perioperatives Outcome, Histologie.

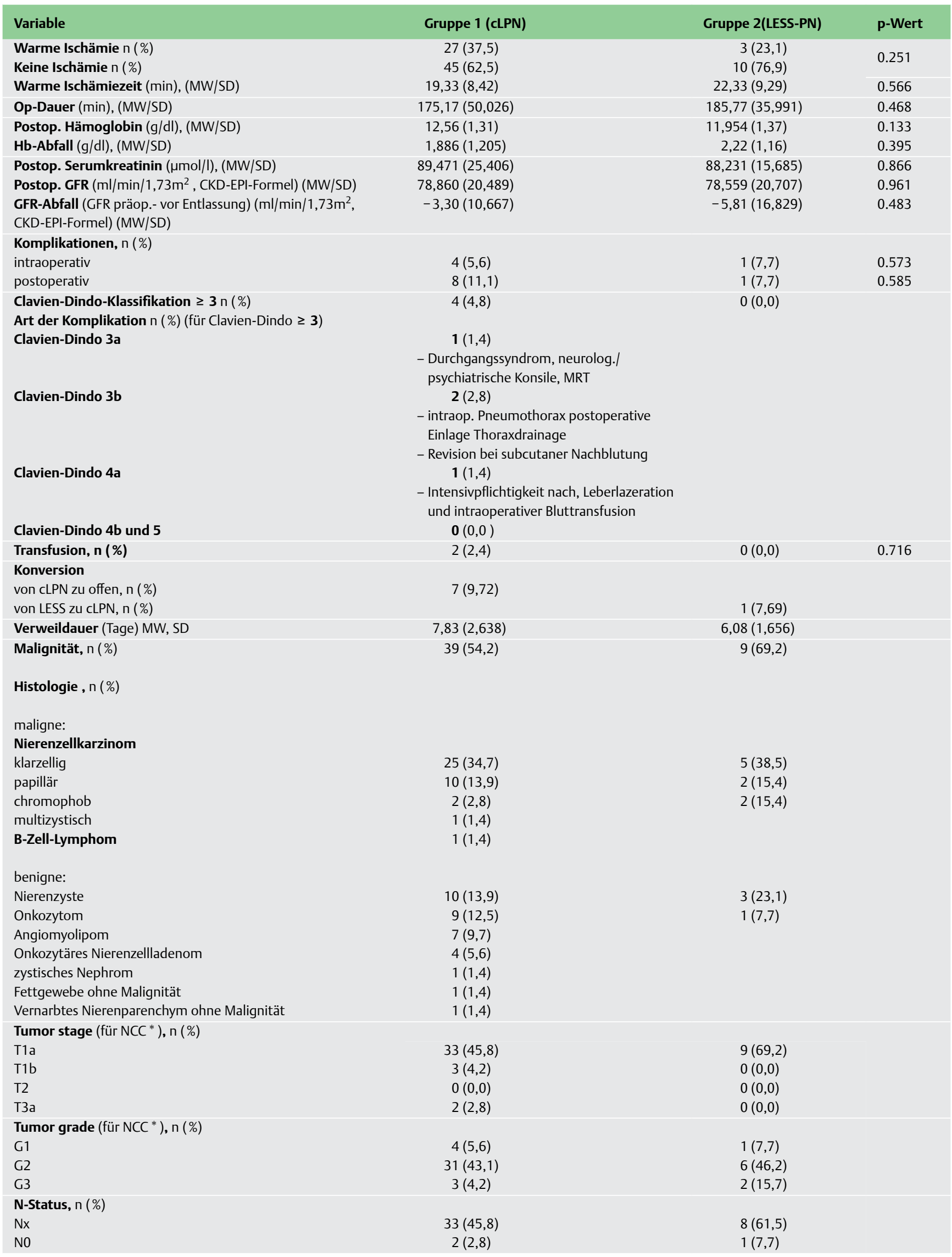


Tab. 2 Fortsetzung.

$\begin{array}{lcc}\text { Variable } & \text { Gruppe } \mathbf{1} \text { (cLPN) } & \text { Gruppe 2(LESS-PN) } \\ \text { M-Status, } \mathrm{n}(\%) & 72(100) & 9(100) \\ \text { M0 } & 0(0,0) & 0(0,0) \\ \text { M1 } & & 0(0,0) \\ \text { R-Status (für NCC }{ }^{*} \text { ), } \mathrm{n}(\%) & 1(2,6) & 9(100) \\ \text { Rx } & 35(92,1) & 0(0,0) \\ \text { R0 } & 2(5,3) & 0(0,0) \\ \text { R klinisch vergeben } & 0(0,0) & \end{array}$

* TNM UICC 2009 Klassifikation für Nierenzellkarzinome (NCC)

resektion mit der LESS Nierenteilresektion in SITUS-Technik zeigt, dass für keinen der untersuchten perioperativen Outcomeparameter signifikante Unterschiede zwischen beiden Patientengruppen bestehen.

Während zahlreiche Studien zur konventionellen laparoskopischen Nierenteilresektion durchgeführt wurden, ist die Zahl der Veröffentlichungen über LESS Nierenteilresektionen begrenzt. Bei der Mehrzahl der Untersuchungen handelt es sich zudem um sehr kleine Patientenserien oder retrospektive multiinstitutionelle Studien. Im Jahr 2011 veröffentlichten Kaouk et al. eine große multiinstitutionelle Studie, bei der 127 LESS partielle Nephrektomien eingeschlossen wurden. Die Operationszeit betrug im Durchschnitt 208,3 $\pm 165,3 \mathrm{~min}$, die warme Ischämiezeit wurde mit 18,4 $\pm 15,5 \mathrm{~min}$ angegeben [21]. Diese Zahlen entsprechen weitgehend den Ergebnissen unserer Untersuchung. In einer weiteren multiinstitutionellen Studie von Greco et al. 2013 wurden bei 190 Patienten Nierenteilresektionen in LESS-Technik durchgeführt. In diesem Patientenkollektiv betrug die mittlere Tumorgröße 2,7 cm, der durchschnittliche PADUA Score wurde mit 7,2 angegeben [22]. Die mittlere Tumorgröße unserer Patienten von 2,68 $\pm 1,47$ (Gruppe 1) bzw. 2,46 $\pm 1,11$ (Gruppe 2) sowie PADUA Scores von $6,86 \pm 0,89$ (Gruppe 1) und 7,46 $\pm 0,97$ (Gruppe 2) stimmen mit den von Greco publizierten Ergebnissen überein. Greco und Kollegen berichten, dass $63 \%$ der Patienten mit warmer Ischämie operiert wurden, wobei die durchschnittliche Ischämiezeit bei $16,5 \mathrm{~min}$ lag [22]. In unserer Studie wurden lediglich $37,5 \%$ der konventionellen und $23,1 \%$ der LESS-Nierenteilresektionen unter Abklemmung der Nierengefäße durchgeführt. Die warme Ischämiezeit betrug dabei 19,3 min (Gruppe 1) bzw. 22,3 min (Gruppe2). Choi et al. berichteten 2011 von 171 Patienten von denen 94,9\% eine roboter-assistierte Nierenteilresektion in LESS-Technik erhielten. Die mittlere Ischämiezeit betrug bei $29 \mathrm{~min}$ [23]. Vor diesem Hintergrund lässt sich feststellen, dass die Ergebnisse unserer Studie im Bereich der publizierten Untersuchungen liegen.

Auch im Vergleich der intra-und postoperativen Komplikationsraten zeigten sich in unserer Studie keine signifikanten Unterschiede zwischen beiden Patientengruppen ( $\bullet$ Tab. 2). In einer Studie von Wang et al. aus dem Jahr 2014 traten nach 21,4\% der Operationen Komplikationen auf [24]. Greco et al. verzeichneten bei $14,7 \%$ der Patienten postoperative Komplikationen [22]. Die von uns ermittelten postoperativen Komplikationsraten lagen hingegen bei 11,1\% (cLPN) und 7,7\% (LESS-PN).

Zusammenfassend stellen wir fest, dass bei keinem der untersuchten Parameter signifikante Unterschiede bezüglich des klinischen Outcomes zwischen beiden Patientengruppen bestanden.
Unsere Ergebnisse bilden die gesamte anfängliche Lernkurve, der an der Medizinischen Hochschule Hannover konventionell laparoskopisch sowie in LESS-Technik durchgeführten Operationen seit Etablierung der minimalinvasiven Nierenteilresektion ab. Die Anzahl der minimalinvasiv operierten Patienten steigt stetig an, so dass sich vor allem im maturen Abschnitt der Lernkurve (ab 2014) die Operations- und Ischämiezeiten deutlich verkürzt haben. Weitere Studien, mit dem Ziel der Abbildung dieser Lernkurve sind erforderlich um den aktuellen klinischen Standard wiederzuspiegeln. Die relativ geringe Anzahl der eingeschlossenen Patienten ist neben dem retrospektiven Charakter ein Schwachpunkt unserer Untersuchung.

Aus unserer Erfahrung und den hier dargestellten Ergebnissen lassen sich die Aussagen von Choi et al. und Protretzke et al. nicht unmittelbar nachvollziehen, die in einem kürzlich erschienenen Review-Artikel die Überlegenheit der robotisch assistierten Nierenteilresektion gegenüber der konventionellen laparoskopischen Nierenteilresektion betonten $[25,26]$. Für Tumore mit niedrigen bzw. mittleren PADUA und RENAL Scores sind die dargestellten Verfahren konkurrenzfähig. Für komplexere Tumore sind sicherlich die operativen Vorteile der Dexterität der robotisch assistierten Laparoskopie/Laparoendoskopie von Vorteil.

Jedoch können wir die Feststellung der Europäischen Guidelines für Robotic and LESS Chirurgie von 2013 unterstreichen, in denen darauf hingewiesen wird, dass die laparoskopische wie laparoendoskopische partielle Nephrektomie ein Feld für erfahrene Laparoskopeure ist [27].

Die Tatsache, dass die histologische Untersuchung bei $45,8 \%$ der Patienten in Gruppe 1 (Tumordiameter 2,68 $\pm 1,47 \mathrm{~cm}$ ) einen benignen Befund ergab, unterstreicht die Feststellung der EAU Leitlinien für Nierenzellkarzinome, dass die partielle Nephrektomie das Standardverfahren für die Behandlung von pT1 Tumoren darstellt. Eine Veröffentlichung von Frank et al. aus dem Jahr 2003 ergab, dass ca. $50 \%$ der Tumoren $<1 \mathrm{~cm}$ benigne sind, mit zunehmender Größe steigt der Anteil maligner Befunde deutlich an [28]. Der hohe Anteil benigner Tumoren in unserer Studie ist ein weiterer Grund dafür, bei kleinen Nierentumoren ein minimalinvasives Verfahren zu favorisieren, sofern dies möglich ist. Nach unserem heutigen Algorithmus werden im klinischen Alltag vor allem die komplexen Fälle mit ungünstig gelegenen Tumoren als offen-chirurgische oder konventionell laparoskopische Operationen durchgeführt. Die Indikation für Nierenteilresektionen in LESS-Technik wird streng unter Berücksichtigung von PADUA und RENAL Score gestellt, insbesondere bei Patienten, für die Kosmetik eine wichtige Rolle bei der Wahl des operativen Zugangsweges spielt. 


\section{Schlussfolgerung}

$\nabla$

Die LESS Nierenteilresektion in SITUS-Technik ist eine attraktive Alternative zur konventionellen laparoskopischen Nierenteilresektion und zur offenen partiellen Nephrektomie. Sie ermöglicht erfahrenen Laparaskopeuren ein Operieren unter den gewohnten Bedingungen und kann vor allem Patienten angeboten werden, die besonderen Wert auf ein nahezu narbenfreies kosmetisches Ergebnis legen.

Interessenkonfilkt: Thomas R. W. Herrmann, Florian Imkamp, Udo Nagele und Markus. A. Kuczyk sind Company Consultants für Karl Storz GmbH, Tuttlingen, Deutschland.

\section{Literatur}

1 Ljungberg B, Bensalah $K$, Canfield $S$ et al. EAU guidelines on renal cell carcinoma: 2014 update. Eur Urol 2015; 67: 913-924

2 Gill IS, Kavoussi LR, Lane BR et al. Comparison of 1800 laparoscopic and open partial nephrectomies for single renal tumors. J Urol 2007; 178: $41-46$

3 Crispen PL, Boorjian SA, Lohse CM et al. Outcomes following partial nephrectomy by tumor size. J Urol 2008; 180: 1912-1917

4 Benway BM, Bhayani SB, Rogers CG et al. Robot assisted partial nephrectomy versus laparoscopic partial nephrectomy for renal tumors: a multi-institutional analysis of perioperative outcomes. J Urol 2009; 182: 866-872

5 Zimmermann R, Janetschek G. Complications of laparoscopic partial nephrectomy. World J Urol 2008; 26: 531-537

6 Gill IS, Kamoi K, Aron M et al. 800 Laparoscopic partial nephrectomies: a single surgeon series. J Urol 2010; 183: 34-41

7 Gill IS, Matin SF, Desai MM et al. Comparative analysis of laparoscopic versus open partial nephrectomy for renal tumors in 200 patients. J Urol 2003; 170: 64-68

8 Georgiou AN, Rassweiler J, Herrmann TR et al. Evolution and simplified terminology of natural orifice transluminal endoscopic surgery NOTES), laparoendoscopic single-site surgery LESS), and mini-laparoscopy ML). World J Urol 2012; 30: 573-580

9 Stolzenburg JU, Kallidonis P, Oh MA et al. Comparative assessment of laparoscopic single-site surgery instruments to conventional laparoscopic in laboratory setting. J Endourol 2010; 24: 239-245

10 Nagele U, Walcher U, Herrmann TR. Initial experience with laparoscopic single-incision triangulated umbilical surgery SITUS in simple and radical nephrectomy. World J Urol 2012; 30: 613-618

11 Schoenthaler M, Avcil T, Sevcenco S et al. Single-Incision Transumbilical Surgery SITUS versus Single-Port Laparoscopic Surgery and conventional laparoscopic surgery: a prospective randomized comparative study of performance with novices in a dry laboratory. World J Urol 2014; 33: 51-57
12 Wolters M, Imkamp F, Wohlatz L et al. Laparoendoscopic Single-Incision Triangulated Umbilical Surgery for Partial Nephrectomy: Early Experience in the First 13 Cases. Journal of Endourology Part B, Videourology 2015; 29: doi:10.1089/vid.2014.0049

13 Wolters $M$, Imkamp F, Wohlatz L et al. Laparoendoscopic partial nephrectomy in single-incision triangulated umbilical surgery SITUS technique: early experience. World J Urol 2015; 33: 403-412

14 Imkamp F, Kuczyk M, Nagele U et al. Single-Incision Pyeloplasty - As Feasible As Laparoscopic Pyeloplasty? Results of the Initial Nine Cases. Journal of Endourology Part B, Videourology 2013; 27: doi:10.1089/ vid.2012.0035

15 Horstmann M, Kugler M, Anastasiadis AG et al. Laparoscopic radical cystectomy: initial experience using the single-incision triangulated umbilical surgery SITUS technique. World J Urol 2012; 30: 619-624

16 Kutikov A, Uzzo RG. The R.E.N.A.L. nephrometry score: a comprehensive standardized system for quantitating renal tumor size, location and depth. J Urol 2009; 182: 844-853

17 Ficarra $V$, Novara G, Secco $S$ et al. Preoperative aspects and dimensions used for an anatomical PADUA classification of renal tumours in patients who are candidates for nephron-sparing surgery. Eur Urol 2009; 56: 786-793

18 Dindo D, Demartines N, Clavien PA. Classification of surgical complications: a new proposal with evaluation in a cohort of 6336 patients and results of a survey. Ann Surg 2004; 240: 205-213

19 Mitropoulos D, Artibani W, Graefen $M$ et al. Reporting and grading of complications after urologic surgical procedures: an ad hoc EAU guidelines panel assessment and recommendations. Eur Urol 2012; 61: 341-349

20 Weight CJ, Larson BT, Fergany AF et al. Nephrectomy induced chronic renal insufficiency is associated with increased risk of cardiovascular death and death from any cause in patients with localized cT1b renal masses. J Urol 2010; 183: 1317-1323

21 Kaouk JH, Autorino R, Kim FJ et al. Laparoendoscopic single-site surgery in urology: worldwide multi-institutional analysis of 1076 cases. Eur Urol 2011; 60: 998-1005

22 Greco F, Autorino R, Rha KH et al. Laparoendoscopic single-site partial nephrectomy: a multi-institutional outcome analysis. Eur Urol 2013; 64: 314-322

23 Choi KH, Ham WS, Rha KH et al. Laparoendoscopic single-site surgeries: a single-center experience of 171 consecutive cases. Korean J Urol 2011; 52: 31-38

24 Wang H, Zhou L, Guo J et al. Mini-flank supra- $12^{\text {th }}$ rib incision for open partial nephrectomy compared with laparoscopic partial nephrectomy and traditional open partial nephrectomy. PLoS One 2014; 9: e89155

25 Choi JE, You JH, Kim DK et al. Comparison of perioperative outcomes between robotic and laparoscopic partial nephrectomy: a systematic review and meta-analysis. Eur Urol 2015; 67: 891-901

26 Potretzke AM, Bhayani SB. Laparoscopic partial nephrectomy: rest in peace. Eur Urol 2015; 67: 902-903

27 Merseburger AS, Herrmann TR, Shariat SF et al. EAU guidelines on robotic and single-site surgery in urology. Eur Urol 2013; 64: 277-291

28 Frank I, Blute ML, Cheville JC et al. Solid renal tumors: an analysis of pathological features related to tumor size. J Urol 2003; 170: 2217-2220 